\title{
Identifying thyroid follicular proliferations that need lobectomy; value of the Bethesda category 4
}

\author{
Kankanamge A, Liyanage TG, Mudduwa LKB \\ Department of Pathology, Faculty of Medicine, University of Ruhuna, Galle, Sri Lanka.
}

Correspondence: Dr. Thusharie Liyanage

e-mail: thusharieliyanage@yahoo.com

(D) https://orcid.org/0000-0001-5276-5054

Submitted on 07.05.2021 and accepted for publication on 05.06.2021

\begin{abstract}
Introduction: Thyroid follicular proliferations (TFPs) identified on fine needle aspiration cytology can be either follicular lesions of undetermined significance; Bethesda category 3 or follicular neoplasms and lesions suspicious for a follicular neoplasm; Bethesda category 4 . The purpose of categorizing to Bethesda category 4 is to identify a thyroid nodule that might be a follicular carcinoma and triage it for thyroid lobectomy. This study was designed to determine the predictive value of categorizing TFPs to Bethesda category 4 in deciding on lobectomy for cytologically identified TFPs.

Methods: This retrospective study included all patients with TFPs identified on cytology, and had a subsequent histological diagnosis, at our unit, over a period of two years. TFPs were categorized into either Bethesda category 3 or 4 on cytology, according to the Bethesda system. Sensitivity, specificity and positive (PPV) and negative predictive values (NPV) for Bethesda category 4 in identifying neoplastic TFPs and implied risk of malignancy for Bethesda category 3 and 4 were calculated taking histopathology as the gold standard. A total of 52 TFPs (Bethesda category 3; $n=39$, Bethesda category 4; $n=13$ ) were included. Subsequent histology has confirmed 18 neoplastic and 34 non-neoplastic TFPs. Sensitivity and specificity of Bethesda category 4 in identifying neoplastic TFPs were $66.66 \%$ and $97.05 \%$ respectively. PPV and NPV of Bethesda category 4 were $92.3 \%$ and $84.6 \%$ respectively. Implied risk of malignancy for Bethesda category 3 and 4 were $5.12 \%$ and $15.38 \%$ respectively.
\end{abstract}

Conclusions: Bethesda category 4 predicts the TFPs that need thyroid lobectomy to triage for follicular carcinoma. The Bethesda category 3 and 4 carry an implied risk of malignancy comparable to the expected values given in Bethesda system.

Key Words: Bethesda system, cytology, follicular proliferations, neoplastic and non-neoplastic lesions, thyroid.

\section{Introduction}

Thyroid nodules are frequently encountered neck lumps in surgical pathology practice, which can either be neoplastic or non-neoplastic. A neoplastic thyroid enlargement could be either benign or malignant. The common benign non-neoplastic conditions of the thyroid gland, sometimes presenting as nodules are, dominant nodule of multinodular goitre, thyroiditis, colloid cysts, hyperplastic nodules and Grave's disease. Neoplastic lesions can be benign adenomas and differentiated or undifferentiated carcinomas (1). Patients with these nodules usually present with clinically significant palpable nodules or they can be incidental findings in radiological examination. 
Fine Needle Aspiration Cytology (FNAC) plays a pivotal role in the diagnosis of these thyroid lesions, and it has been identified as the method of choice among other modes of investigations (2). FNAC is accepted as an accurate, relatively inexpensive and rapid to diagnose thyroid lesions preoperatively. Its overall sensitivity and specificity are reported to be $95 \%$ and $98 \%$ respectively (3).

By using adequately sampled, well prepared smears, diffuse changes such as thyroiditis, colloid goitres as well as malignant lesions like papillary carcinoma, medullary thyroid carcinoma, anaplastic carcinoma and thyroid lymphoma can often be diagnosed. However, the pathologist has to be accurate in the preoperative cytological diagnosis of follicular lesions as the surgical management is different for the non-neoplastic and neoplastic proliferations. Final confirmation of the diagnosis of follicular carcinoma and adenoma are solely dependent on histological identification of capsular or vascular invasion following surgery (4). In addition, the presence of several differential diagnoses for the follicular cytoarchitectural pattern results in problems in interpreting follicular pattern in cytology. The various types of follicular lesions encountered in thyroid cytology have variable risk of malignancy. These include; hyperplastic nodules, follicular or Hürthle cell adenomas, follicular variant papillary thyroid carcinoma and the recently described indolent lesion; Non-Invasive Follicular Tumour with Papillary-like nuclear features (NIFTP) (4).

Although, it seems that accurate preoperative cytological diagnosis of a follicular lesion is a debatable issue, the cytoarchitectural details seen in a smear can be used as a screening test for follicular lesions of the thyroid. In this regard, the necessity for a standardized reporting system was felt extremely important, and the THY system has been used for this purpose up to 2017 in Sri Lanka.

According to the THY system, when the fine needle aspiration cytology of thyroid shows follicular pattern, it was diagnosed as Thy3 category i.e. follicular proliferation. Most of the pathologists in our setting used to favour the pattern of proliferation towards either a non-neoplastic process or a follicular neoplasm. However, there were no accepted uniform criteria to differentiate a follicular neoplasm from non- neoplastic lesion in the THY 3 category (5). This was agreed to be a major deficiency in the THY system as further management of these two types of follicular lesions are completely different. To address this deficiency, The Bethesda System in reporting thyroid cytopathology was introduced and it was adopted in reporting thyroid fine needle aspirations in our country too (6).

The Bethesda system identifies six diagnostic categories (6). The category 1 includes nondiagnostic and unsatisfactory aspirates for reporting or cyst fluid only. The category 2 includes all benign thyroid lesions like colloid nodules, autoimmune thyroiditis, lymphocytic thyroiditis and granulomatous thyroiditis. Bethesda category 3 indicates atypia of undetermined significance or follicular lesion of undetermined significance. Bethesda category 4 includes all follicular neoplasms or suspicious for follicular neoplasm. Lesions which are suspicious for malignancy are included in the Bethesda category 5. All thyroid lesions with unequivocal cytological evidence of malignancy like papillary carcinoma, medullary carcinoma, anaplastic carcinoma, squamous cell carcinoma, metastatic carcinomas and lymphomas are included in the category 6.

In the Bethesda system, the category 3 and 4 are the newly introduced categories for thyroid cytology reporting in order to meet the aforementioned deficiency in the THY system (6). The Bethesda category 3 which denotes atypia of undetermined significance or the follicular lesion of undetermined significance recommends two management options, that is to follow up or to repeat FNAC. The category 4 which include follicular neoplasms or lesions suspicious of follicular neoplasm recommends lobectomy as the management (7). Moreover, there are standard criteria stated in the Bethesda system to categorize a follicular cytological pattern to either Bethesda category 3 or 4 (8).

Thus, adopting the Bethesda System in reporting thyroid cytopathology is expected to be helpful in better management of patients by improving preoperative identification of neoplastic follicular lesions which need lobectomy to identify possible follicular carcinoma (9). 
Therefore, this study was designed to determine the validity of the Bethesda system in cytological differentiation of follicular neoplasms from nonneoplastic follicular lesions and to assess the risk of malignancy by each of the two categories; Bethesda 3 and 4.

\section{Methods}

This retrospective study was carried out in the Department of Pathology, Faculty of Medicine, University of Ruhuna, Galle. This study included patients who were diagnosed to have follicular proliferations on cytology, either belonging to Thy 3 category or Bethesda 3/4 categories at the Department of Pathology, Teaching Hospital Karapitiya and the Department of Pathology, Faculty of Medicine, University of Ruhuna. All patients subsequently underwent surgical management and received a histopathology report. Patients who were diagnosed from January 2017 to December 2018 were recruited for the study.

Patients who did not undergo surgical management for the cytologically detected follicular thyroid lesions and have not been issued with a histopathology report were excluded. Cases of Bethesda Category 3 (with no Thy 3 categorization) which were categorized into Bethesda 3 based on the presence of atypia without follicular proliferations were also excluded.

Smears from all cases of follicular proliferations were reassessed. The smears with faded staining were re-stained for reassessment. The reassessment was done by the first and the second authors independently and categorized them into either Bethesda category 3 or 4 according to the standard criteria for the Bethesda categorization (8). (Figures $1-4)$.

Any discrepancy between the two assessors were sorted by examining under a multi-head microscope arriving at a consensus on the Bethesda category. Both assessors were blinded to the original cytological and the histological diagnosis. Finally, the comparison between reclassified cytological cases and their relevant histological diagnosis (the gold standard) was done. On comparison, histology of the relevant lesion which was subjected to FNAC, was considered for the analysis and pathology of the rest of the specimen was assessed as background pathology only.

By correlating with histopathology, sensitivity, specificity and positive (PPV) and negative predictive values (NPV) for Bethesda Category 4, in identifying neoplastic TFPs and implied risk of malignancy for Bethesda Category 3 and Bethesda Category 4 were calculated.

Ethical approval for the study has been obtained from Ethical Review Committee, Faculty of Medicine, University of Ruhuna.

\section{Results}

Seventy patients with cytologically diagnosed follicular proliferations in the thyroid gland were identified in these two centers during the period of two years. Histopathology reports were available only in 48 patients. As there were 4 patients within the total 48 patients with bilateral thyroid nodules and they had received separate cytology reports for those nodules as follicular proliferations at different times, every single clinical nodule was counted separately. Finally, a total of 52 cases of follicular proliferation in the thyroid gland were included. Among them, 39 cases $(75 \%)$ were diagnosed as Bethesda category 3 and the other 13 cases $(25 \%)$ were diagnosed as Bethesda category 4.

Subsequent histology has confirmed 18 neoplastic and 34 non-neoplastic thyroid follicular proliferations (Table 1). Comparison of the Bethesda category and the corresponding histological pattern of the follicular lesion is shown (Table 2).

These results were analysed to assess the validity of the Bethesda system in cytological differentiation of neoplastic and non-neoplastic follicular lesions. In order to assess the validity; sensitivity, specificity, positive predictive value and the negative predictive value of Bethesda 4 category in identifying neoplastic follicular lesions were calculated (Table 3).

Sensitivity of Bethesda category 4 was $66.66 \%$ and the specificity was $97.05 \%$. Positive predictive value was $92.3 \%$ and negative predictive value was $84.6 \%$. Implied risk of malignancy for Bethesda category 3 and Bethesda category 4 were $5.12 \%$ $(2 / 39 \times 100)$ and $15.38 \%(2 / 13 \times 100)$ respectively. 


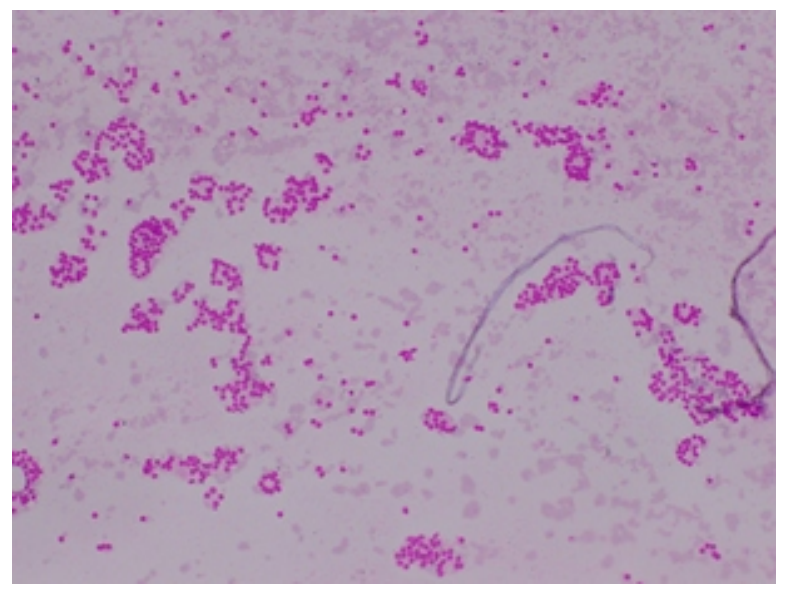

Figure 1: Follicular proliferation in cytology Bethesda category 3 (Leishman stain, $\mathrm{x} 40$ )

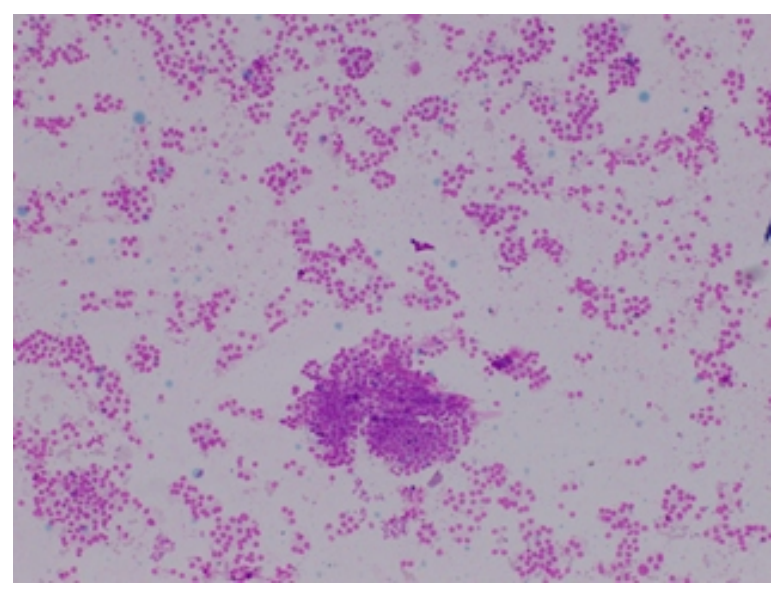

Figure 3: Follicular proliferation in cytology Bethesda category 4 (Leishman stain, $\mathrm{x} 40$ )

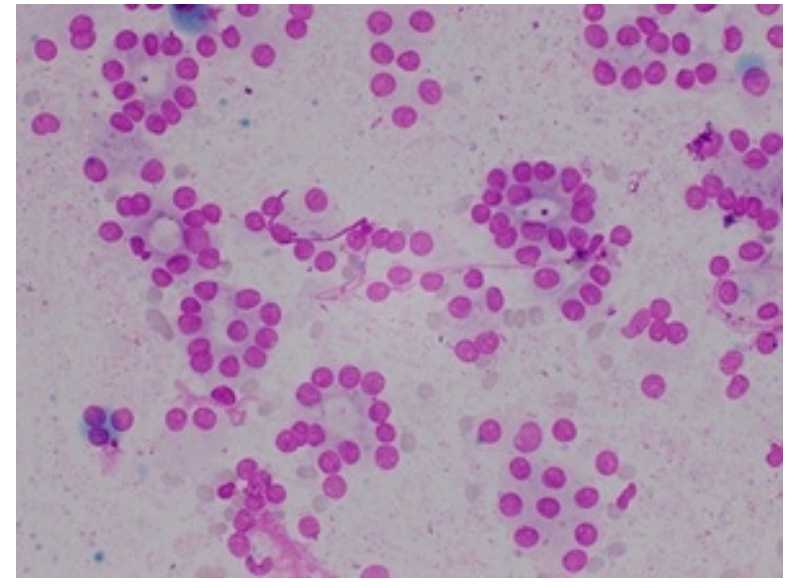

Figure 2: Follicular proliferation in cytology Bethesda category 3 (Leishman stain, $\mathrm{x}$ 400)

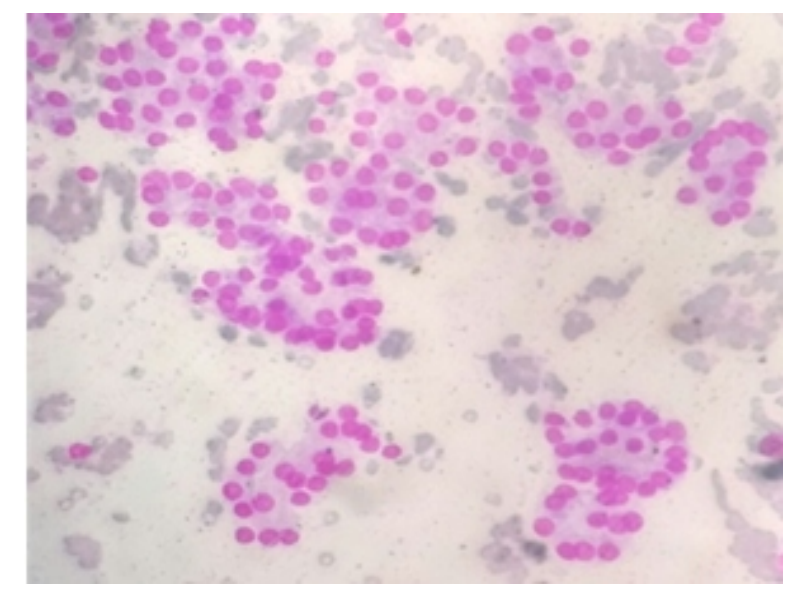

Figure 4: Follicular proliferation in cytology Bethesda category 4 (Leishman stain, $\mathrm{x}$ 400)

Table 1: Comparison of the Bethesda category and corresponding histology (non-neoplastic and neoplastic status of the follicular lesion on histology)

\begin{tabular}{lccc}
\hline \multirow{2}{*}{ Histopathology } & \multicolumn{2}{c}{ Bethesda Category } & \multirow{2}{*}{ Total } \\
\cline { 2 - 3 } & Bethesda 3 & Bethesda 4 & \\
\hline Neoplastic & 6 & 12 & 18 \\
& $15.4 \%$ & $92.3 \%$ & $34.6 \%$ \\
Non-neoplastic & 33 & 1 & 34 \\
& $84.6 \%$ & $7.7 \%$ & $65.4 \%$ \\
\hline Total & 39 & 13 & 52 \\
\hline
\end{tabular}


Table 2: Comparison of Bethesda category and corresponding histological diagnosis of follicular lesions

\begin{tabular}{lccc}
\hline \multirow{2}{*}{ Histopathology } & \multicolumn{2}{c}{ Bethesda Category } & \multirow{2}{*}{ Total } \\
\cline { 2 - 3 } & Bethesda 3 & Bethesda 4 & \\
\hline Hyperplastic nodule & 18 & 1 & 19 \\
& $46.2 \%$ & $7.7 \%$ & $26.9 \%$ \\
Autoimmune thyroiditis & 15 & 0 & 15 \\
Adenoma & $38.5 \%$ & $0.0 \%$ & $28.8 \%$ \\
Carcinoma (follicular carcinoma, Hürthle cell & 4 & 10 & 14 \\
carcinoma, Follicular variant papillary carcinoma) & $10.3 \%$ & $76.9 \%$ & $26,4 \%$ \\
& 2 & 2 & 4 \\
\hline
\end{tabular}

Table 3: Assessment of the validity of Bethesda Category 4 in identifying neoplastic follicular lesions

\begin{tabular}{lccc}
\hline Cytology & \multicolumn{3}{c}{ Gold standard - Histology } \\
\cline { 2 - 4 } & Positive for Neoplasm & Negative for Neoplasm & Total \\
\hline Bethesda Category 4 & True positives & False positives & \\
Positive & 12 & 1 & 13 \\
Bethesda category 4 & False negatives & True negatives & 39 \\
Negative & 6 & 33 & \\
\hline Total & 18 & 34 & 52 \\
\hline
\end{tabular}

\section{Discussion}

This study was carried out to investigate the value of Bethesda system on reporting thyroid cytology in deciding on the surgical intervention for cytologically identified follicular proliferations in thyroid gland in a tertiary care hospital. The current study sample of 52 follicular proliferations had $75 \%$ cases of Bethesda category 3 and $25 \%$ of Bethesda category 4 . This finding was comparable with the findings of other studies, where cohort incidence rate of Bethesda 3 category was 4 times higher than Bethesda category 4 ( $8 \%$ and $2 \%$ respectively) (10).

The calculated sensitivity and specificity of Bethesda category 4 were, $66.66 \%$ and $97.05 \%$. respectively. Further, Bethesda category 4 criteria has $97.05 \%$ specificity in excluding non- neoplastic follicular proliferations on cytology. Similarly, the positive and negative predictive value in identifying neoplastic follicular lesion by Bethesda category 4 were $92.3 \%$ and $84.6 \%$ respectively. This also explains that Bethesda category 4 criteria can specifically identify neoplastic follicular lesions in cytology, and there is $92 \%$ predictability of a lesion becoming neoplastic when a smear is assigned to Bethesda category 4.

In the same way, by looking at the cytology smear of a follicular lesion, a pathologist can predict with $92 \%$ probability that the lesion cannot be a neoplastic follicular lesion. That is, when a smear was categorized into Bethesda category 3 , only 8 out of 100 cases need surgical intervention and remainder needs only the follow-up. This prevents unnecessary surgical intervention in patients with non-neoplastic thyroid follicular lesions.

The implied risk of malignancy for Bethesda category 3 was $5.12 \%$ and that for Bethesda category 
4 was $15.38 \%$. These figures are comparable to the study done by Mosca et al., and the Bethesda guidelines $(6,11)$.

Similarly, several studies have concluded that the implied risk of malignancy for Bethesda category 4 to be between 15 to $30 \%(12,13)$. Although, our value complies with those results, it occupies the lower limit of the reported range. This might be due to the small size of the sample assessed in our study. However, these results clearly explain that the lesions of thyroid having cytologically detected follicular proliferation, categorized into Bethesda category 3 has very low risk of malignancy than those assigned to Bethesda category 4 and need for surgical intervention are comparatively lower in former than for the latter (10). The cases which are cytologically assigned to Bethesda category 3 , usually need no surgical intervention but require follow up as recommended by the Bethesda system of reporting. However, cases which are cytologically classified into Bethesda category 4, need surgical intervention (lobectomy) to assess whether there is capsular or vascular invasion.

\section{Conclusions}

Our study highlights the value of Bethesda 3 and 4 in categorization of cytologically identified TFPs in triaging thyroid lesions for lobectomy. Bethesda category 4 has sufficient predictability in identifying TFPs that need thyroid lobectomy to triage for follicular carcinoma. The Bethesda 3 and 4 carry an implied risk of malignancy comparable to the values stated for Bethesda system.

\section{Acknowledgements}

The staff of Department of Pathology, Faculty of Medicine, University of Ruhuna and staff of Department of Pathology, Teaching Hospital Karapitiya.

\section{References}

1. Rosai, J., Giovanni. Thyroid Gland. In: J. Rosai, Rosai \& Ackerman's Surgical Pathology. Chapter 9. Missouri: Elsevier: 2011.
2. Hirachand S, Maharjan M, Lakhey M, Thapa R, Kafle S. Accuracy of fine needle aspiration cytology in diagnosis of thyroid swelling. Nep-JOL. 2013; 3: 433-436.

3. Alwahaibi N, Alsalami J, Bai UR, Lakhtakia R. Accuracy of the fine needle aspiration cytology of thyroid lesion with corresponding histopathology: A single institution experience. Thyroid Res Pract. 2016; 13: 140-143.

4. Faquin WC. Diagnosis and Reporting of Follicular Patterned Thyroid Lesions by Fine Needle Aspiration. Head and Neck Pathol. 2009; 3: 82-85.

5. Seneviratne B. Interpretation of fine needle aspiration cytology of thyroid: do we need a better classification. SJDEM. 2012;2(3): 32-34.

6. Cibas ES, Ali SZ. The 2017 Bethesda System for Reporting Thyroid Cytopathology. Thyroid. 2017; 27(11): 13411346.

7. Jeffrey FK Nayar R, Andrew A, Renshaw. Atypia of Undetermined Significance/Follicular Lesion of Undetermined Significance. In: Ali SZ, Cibas ES (eds). The Bethesda System for Reporting Thyroid Cytopathology. Definitions, Criteria and Explanatory Notes. Chapter 4. London: Springer; 2007.

8. Henry MR, DeMay RM, Berezowski K. Follicular Neoplasm/ Suspicious for a Follicular Neoplasm. In: Ali SZ, Cibas ES (eds). The Bethesda System for Reporting Thyroid Cytopathology. Definitions, Criteria and Explanatory Notes. Chapter 5. London: Springer; 2007.

9. Bukhari MH, Khan AA, Niazi S, Arshad M, Akhtar ZM, et al. Better Thyroid Cytopathology Reporting System May Increase the Clinical Management and Patient Outcome. $J$ Cytol Histol. 2012 Oct; 3: 158.

10. Ho SH, Sarti EE, Jain KS, Wang H, Nixon LJ, Shaha AR, et al. Malignancy Rate in Thyroid Nodules Classified as Bethesda Category III (AUS/FLUS). Thyroid. 2014; 24(5): 832-839.

11. Mosca L, Silva LFFD, Carneiro PC, Chacon DA, AraujoNeto VJFDA, Araujo-Filho VJFD, et al. Malignancy rates for Bethesda III subcategories in thyroid fine needle aspiration biopsy (FNAB). Clinics. 2018; 73: e370.

12. Lewis CM, Chang KP, Pitman M, Faquin WC, Randolph GW. Thyroid fine needle aspiration biopsy: variability in reporting. Thyroid. 2009; 19: 717-723.

13. Sengupta M, Das I, Basu K, Das C, Ara Z, Islam N. A Cytohistological correlation in Thyroid Swelling with Special Reference to The Bethesda System: A Study of 192 Cases. IOSR-JDMS. 2017; 16(10): 55-59. 